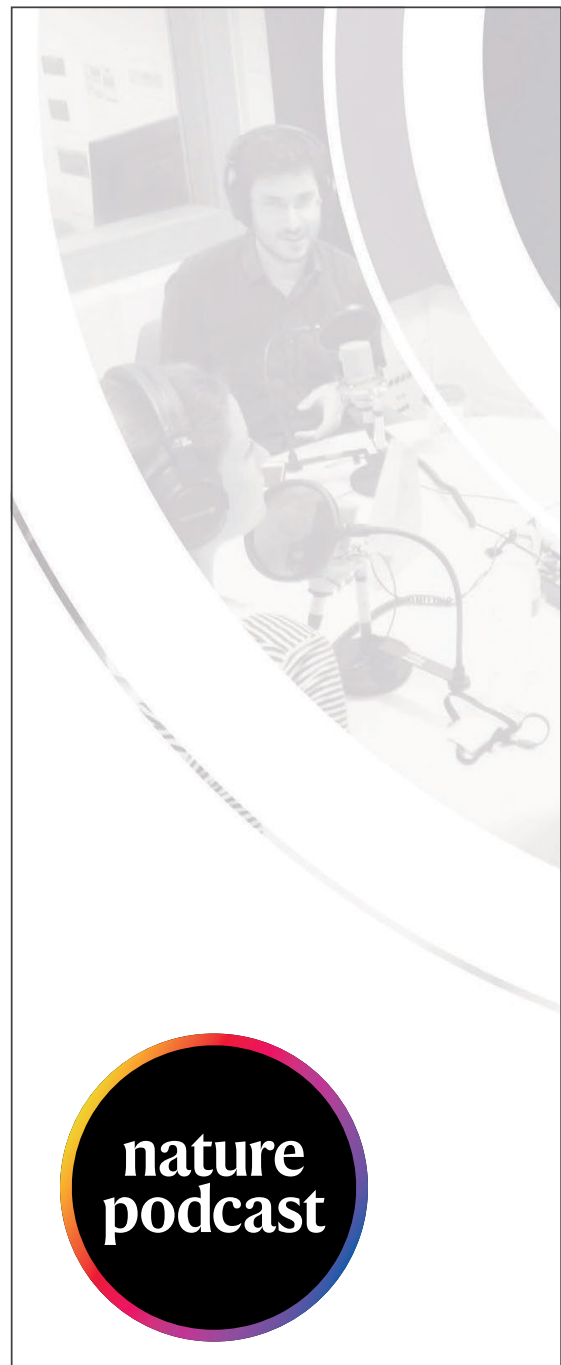

\section{The week's}

best science,

from the world's

leading science

journal.

NATURE.COM/NATURE/PODCAST

\title{
Sensing proteinogenic amino acids
}

Aerolysin nanopores offer a way to identify natural amino acids and their chemical modifications.

$\mathrm{T}$ he rapid advance in nanopore sensing has stimulated research interest in single-molecule protein sequencing, which has been hindered by the technical challenges in differentiating the subtle differences among amino acids. "The development of a fast and low-cost nanopore-based DNA sequencing device prompted my interests in nanopore-based protein sequencing," says Abdelghani Oukhaled from Université Cergy-Pontoise Paris-Seine (CYtech), France.

Nanopore-based sensing enables a direct readout of chemical structure and identity of an analyte through the nanopore ionic current or transverse tunneling current. The amino acid sequence of a peptide, or even a protein, can therefore be recognized. Compared to mass-spectrometry-based methods, nanopore sensing holds great potential in long-read protein sequencing and direct detection of post-translational modifications.

Previously, the Oukhaled group had utilized aerolysin nanopore to distinguish among uniformly charged homopeptides and to assign signature currents to the charged homopeptides (for example, a polycationic peptide). They now chemically linked one amino acid of interest to the polycationic carrier (arginine heptapeptide), allowing transport of the peptide through the nanopore. A transient current blockade is then induced by the transport of the peptide, whereby the characterizations of relative residual current and blockade duration can be used to reveal the identity of the linked amino acid.

Using wild-type aerolysin nanopore, they demonstrate that 13 natural amino acids can be recognized via the distinct current blockades. Surprisingly, the aerolysin nanopore can discriminate amino acids with the same molecular mass, such as leucine and isoleucine, as well as amino acids with similar chemical structures, such as phenylalanine and tyrosine. Oukhaled notes that this is "a resolution which had never been shown before."

To better understand how peptides move through the nanopore, the Aksimentiev group from University of Illinois at

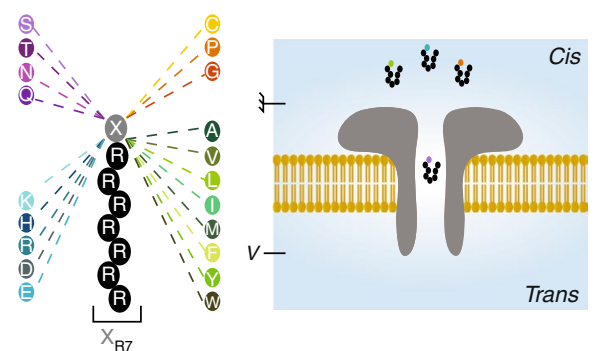

One amino acid of interest $(X)$ is linked to the polycationic carrier for aerolysin nanopore analysis. An external voltage $V$ is applied on the trans side of the bilayer relative to the cis side as ground. Image reprinted with permission from Ouldali, H. et al. Nat. Biotechnol. https://doi. org/10.1038/s41587-019-0345-2 (2019).

Urbana-Champaign used simulation tools to model the nanopore system. The simulation results indicate that an aerolysin nanopore has a built-in single molecule trap that is highly sensitive to the excluded volume of an analyte.

Looking forward, Oukhaled envisions a cleavage-and-ligation approach for protein sequencing, like next-generation sequencing. In this method, a terminal amino acid is cleaved from the target protein and ligated to a polycationic carrier for subsequent nanopore analysis. Jan Behrends from University of Freiburg, whose lab contributed methods for improved discrimination, comments that "it will be very important in the future to address and eliminate the mechanisms that still limit the resolution, especially the electrical noise due to the dynamics of the peptide in the pore. This is an area where, again, simulation and experiment have to go in tandem."

\section{Lei Tang}

Published online: 4 February 2020 https://doi.org/10.1038/s41592-020-0741-z

\section{Research paper}

Ouldali, H. et al. Electrical recognition of the twenty proteinogenic amino acids using an aerolysin nanopore. Nat. Biotechnol. https://doi.org/10.1038/ s41587-019-0345-2 (2019). 